\title{
Inflammation, Microglia and Alzheimer's Disease
}

\author{
Brent Cameron and Gary E. Landreth \\ Alzheimer Research Laboratory Department of Neurosciences Case Western Reserve University \\ Cleveland, $\mathrm{OH} 44106$
}

\begin{abstract}
Microglia are the brain's tissue macrophage and representative of the innate immune system. These cells normally provide tissue maintenance and immune surveillance of the brain. In the Alzheimer's disease brain amyloid deposition provokes the phenotypic activation of microglia and their elaboration of proinflammatory molecules. Recent work has implicated Toll-like receptors in microglial recognition and response to amyloid fibrils. It is now evident that these cells exhibit more complex and heterogeneous phenotypes than previously appreciated that reflect both the plasticity of cells in this lineage and their ability to transition between activation states. The phenotypic diversity is associated with inactivation of the inflammatory response and tissue repair. We discuss recent evidence that the brain can be infiltrated by circulating monocytes in the diseased brain and that these cells may comprise a unique subpopulation of myeloid cells that may be functionally distinct from the endogenous microglia.
\end{abstract}

\section{Keywords}

Microglia; inflammation; Alzheimer's disease; Toll-like receptors; beta amyloid; alternative activation; macrophage; monocyte

\section{Introduction}

The suggestion that inflammation may participate in Alzheimer's disease pathogenesis was first articulated about 20 years ago and despite two decades of work, many of the central questions regarding the inflammatory response in the Alzheimer's disease (AD) brain remain unanswered (Akiyama et al., 2000). In the course of work in this area we have come to a more sophisticated understanding of the role of microglia, the principal immune effector cell in the nervous system, in the diseased brain. The initial work documented the presence of phenotypically activated microglia that were found to surround amyloid deposits in the brain and invest the plaques with their processes. The Alzheimer's brain was also found to have elevated levels of a number of microglial-derived cytokines and other immune mediators, reflective of a chronic inflammatory environment in the $\mathrm{AD}$ brain. It was postulated that this proinflammatory milieu was neurotoxic, and acted to accelerate and exacerbate disease progression, and this remains the prevailing view.

\footnotetext{
(c) 2009 Elsevier Inc. All rights reserved.

Correspondence: Gary Landreth Alzheimer Research Laboratory Department of Neurosciences School of Medicine Case Western Reserve University 10900 Euclid Ave. Cleveland, OH 44106216 368-6101 FAX 216 368-4650 gel2@ case.edu.

Publisher's Disclaimer: This is a PDF file of an unedited manuscript that has been accepted for publication. As a service to our customers we are providing this early version of the manuscript. The manuscript will undergo copyediting, typesetting, and review of the resulting proof before it is published in its final citable form. Please note that during the production process errors may be discovered which could affect the content, and all legal disclaimers that apply to the journal pertain.
} 
An influential review by Wyss-Coray and Mucke (Wyss-Coray and Mucke, 2002) helped catalyze a reevaluation of the roles of microglia in the diseased brain. They argued that the inflammatory response is multifaceted, with both detrimental actions that promote neurodegeneration as well as a spectrum of actions that promote neuronal survival and tissue repair. In the ensuing years, some of the details have been filled in, but investigation of the basic question of the role in microglia-mediated responses in the AD brain have only led to generation of a new array of questions surrounding the biology of this enigmatic cell type. The purpose of this review is to highlight our new insight into the basic physiological actions of microglia and how they are perturbed during the course of Alzheimer's disease. It is our intention to focus on newer controversies in the field. Specifically, how does the activation status of microglia influence their role in pathogenesis or prevention of Alzheimer's disease, new findings on the participation of Toll-like receptors in $\mathrm{AD}$ pathogenesis and the evidence for peripheral monocyte infiltration the brain and their potential roles in mitigating the disease.

\section{Microglia- new insight into the biology of the brain's tissue macrophage}

Microglia are the brain's tissue macrophage (Ransohoff and Perry, 2009). Many of the questions about their role in the AD brain are reliant upon knowledge of the origins and normal behaviors of these cells. Central amongst these questions are whether microglia are resident in the brain for the lifespan of the organism, whether they normally renew themselves through proliferation within the brain, or whether myeloid progenitors normally infiltrate the brain to replace senescent cells. For some of these questions we still only have tentative answers. These questions have largely been of only academic interest until recently when it has been proposed that monocytes in the peripheral circulation immigrate into the mature brain, particularly in the course of neurodegenerative diseases such as $\mathrm{AD}$, which has clear therapeutic implications. The ensuing controversy has now focused attention on the origins of parenchymal microglia in both the developing and mature brain. (Chan et al., 2007)

During development microglial progenitors, derived from mesenchymal myeloid lineage progenitor cells, immigrate into the embryonic brain early in gestation (Chan et al., 2007). They proliferate in situ and exhibit a motile ameboid phenotype, populating the brain before the development and closure of the blood brain barrier. This population is thought to be distinct from fetal macrophages, that are also myeloid in origin but arise from monocytes in the fetal circulation. These fetal macrophages also invade the developing brain and contribute to the microglial population, particularly at later developmental times (Streit et al., 2004). The lack of distinguishing lineage markers has prevented any hard conclusions to be drawn on the relative contribution of these different myeloid cells to the microglia population in the mature brain.

The microglia, like other tissue macrophages, acquire a tissue-specific phenotype that is reflective of local environmental influences. A corollary is that within a single tissue these cells can exhibit a diverse range of phenotypic characteristics, owing the different cellular composition and physiology of a complex tissue such as the brain. Macrophages are extraordinarily plastic and are able to dramatically alter their phenotype and exhibit a remarkable array of behaviors and morphologies in response to environmental stimuli. In the mature brain, the microglia extend ramified processes into the surrounding tissue. Importantly, in the normal brain the interaction of microglia with both neurons and other glia serves to suppress the 'activation' of the cells and dampen their response to proinflammatory signals and spurious induction of an immune response (Colton, 2009). Pairs of receptors that are uniquely expressed by microglia and other neural cell types mediate these interactions. For example, fractalkine is a membrane glycoprotein expressed on the neuronal cell surface and interacts with the chemokine receptor CXCR1 on microglia (Ransohoff et al., 2007). The interaction of these two cell types, during the course of microglial surveillance of the brain, 
acts to suppress microglia activation in the normal brain (Cardona et al., 2006). Neurons also express CD200 on their cell surface that interacts with its corresponding ligand, CD200L, on microglia. The CD200-CD200L interaction acts to inhibit the production of proinflammatory mediators by microglia. CD200 and CD200R levels are reduced in the cortex and hippocampus of AD patients, compared to age matched controls (Walker et al., 2009). It is of particular interest that CD200 expression is positively regulated by the anti-inflammatory cytokine IL-4 (Lyons et al., 2007). There is good evidence that the reciprocal interaction of the receptor pair Signal -Regulatory protein $\alpha$ (SIRP $\alpha$; SHPS-1) and CD47 signals bidirectionally to both cell types. SIRP $\alpha$ is expressed on myeloid cells, astrocytes, and neurons (Adams et al., 1998;

Kusakari et al., 2008) and CD47 is expressed by both microglia and neurons (Matozaki et al., 2009). The effect of neuronal CD47 upon its association with microglial SIRP $\alpha$ is to suppress microglial proinflammatory cytokine expression and inhibit phagocytosis (Matozaki et al., 2009).

Streit and colleagues have argued that in humans (but not rodents) microglia normally senesce and undergo microglial dystrophy, that in some cases involves a process of cytorrhexis (Streit et al., 2004) While this latter process can be observed in normal aging brains, it is more frequently observed in neurodegenerative diseases. Combs has reported that microglia derived from aged animals exhibit a distinct different response to fibrillar $\mathrm{A} \beta$ compared to those from neonates (Floden and Combs, 2006). A recent study examining the behaviors of microglia by two photon microscopy in the living brain of murine models of $\mathrm{AD}$ found that microglia in the aged brain were less motile and possessed fewer processes (Meyer-Luehmann et al., 2008), supporting the view that aging is accompanied by impaired microglial functionality (Streit et al., 2008).

Microglia, in their roles as tissue macrophages, act normally to carry out tissue maintenance and immune surveillance. New insight into the normal biology of microglia has come from advanced imaging methods, allowing direct observation of microglia in the living brain. A series of remarkable studies has revealed that microglia continuously extend and retract processes into the adjacent tissue with a radius of about $80 \mu \mathrm{m}$ (Nimmerjahn et al., 2005). Thus, the entire brain undergoes surveillance by these cells every few hours. The microglia sample their environment by macropinocytic uptake of the extracellular fluid and contact neurons, other glia and vessels. Tissue damage elicits a rapid redirection of microglial processes to the area of damage, and if sustained promotes the migration of the cell to the affected area (Davalos et al., 2005). Microglia also monitor synaptic activity, contacting each synapse about once per hour and acting to remodel functionally impaired synapses (Wake et al., 2009). Analysis of murine models of $\mathrm{AD}$ using these techniques has been quite illuminating, revealing that amyloid plaques form in the brain and become progressively larger, followed by the appearance of microglia that become associated with the plaque and which exhibit an 'activated' phenotype (Yan et al., 2009). Neuritic dystrophy is subsequently observed, although its cause is not known. These findings provide direct evidence that microglia detect and respond to amyloid deposition, which elicits the induction of an inflammatory phenotype. Bornemann et al. have reported that microglia proliferate in the brains of mice with deposited amyloid (Bornemann et al., 2001).

\section{Microglial activation in the AD brain}

There is an extensive literature documenting a microglial-mediated inflammatory response in the AD brain. This topic has been the subject of a number of reviews (Combs, 2009) and we will focus here on recent developments that shed new insight in to the roles of inflammation and microglia in $\mathrm{AD}$ pathogenesis. It should be appreciated that the primary evolutionary pressure on this lineage has been host defense mechanisms, and they are quite efficient in mobilizing these responses. Indeed, microglia and macrophages express a panorama of cell 
surface receptors that they use alone, or in combination, to detect pathogens or foreign materials. Engagement of the host defense mechanisms provokes the conversion of a 'resting' microglia into an 'activated' phenotype that is associated with a robust and coordinated response involving the production of free radicals and the generation and secretion of cytokines, chemokines and acute phase proteins. While this is an effective response for elimination of pathogens, it is maladaptive when these mechanisms are engaged by disease processes that originate within the tissue itself, especially in the CNS. In the AD brain, the formation of amyloid fibrils and their deposition elicits the phenotypic activation of microglia and the panoply of responses that accompany the activation of host-defense mechanisms.

The advent of PET-based techniques that allow imaging of activated microglia in the living human brain has provided a new perspective on the how and when these cells are activated (Cagnin et al., 2007). The availability of carbon 11-labeled compounds that bind to the mitochondrial protein TP-18 (also termed the peripheral benzodiazepine receptor), which is expressed at elevated levels in activated microglia, has allowed direct visualization of phenotypically activated cells. Edison et al. recently reported that they detected an increased abundance of activated microglia in cortex of $\mathrm{AD}$ patients and this increase was not tightly correlated with overall amyloid plaque burden, but rather with reduced cognitive ability (Edison et al., 2008). However, a similar study failed to confirm these results (Wiley et al., 2009). Okello et al. reported that there was a correlation between diminished cognitive status and microglial activation in a subset of patients with mild cognitive impairment (Okello et al., 2009). The sensitivity of the method is limited by the radioligand employed in these studies. New compounds with better sensitivity are now available and may permit more definitive analyses (Doorduin et al., 2009). Analysis of murine models of AD using such a compound revealed a robust correlation of microglial activation and plaque deposition (Maeda et al., 2007). The elevated expression of TP- 18 has also been reported in plaque-associated astrocytes (Ji et al., 2008).

\section{The roles of Toll-like receptors in microglia in the AD brain}

Microglia are the representatives of the innate immune system in the brain and possess a number of receptors, most prominently the Toll-like receptors (TLRs), through which they are able to detect microbial and viral pathogens and tissue damage (Bianchi, 2007; Palm and Medzhitov, 2009). Tissue damage results in the release of intracellular proteins that comprise 'damageassociated molecular pattern' molecules (DAMPs) that are detected by TLRs and other cell surface receptors and elicit an inflammatory response. Microbial pathogens express conserved pathogen-associated molecular patterns (PAMPs) that are common to microbes and allow their rapid and efficient recognition by the innate immune system primarily through TLRs that are specialized to recognize these molecules. Other pattern recognition receptors, which include the mannose receptor (CD206), scavenger receptors, C-type lectins, and their expression by microglia /macrophages reflects the distinctive physiology of these cells. Thus, either infections or injury results in a similar response from the innate immune system. While the innate immune system can effectively ward off invading bacteria, this comes at a cost, as the engagement of the innate immune response results in collateral damage to the tissue.

Most prominent amongst the pattern recognition receptors are the Toll-like receptors (TLRs) that detect bacterial coat proteins or lipids. Of relevance to Alzheimer's disease, TLR4 and TLR2 are essential for defense against Gram-negative and Gram-positive bacteria, respectively. It has only recently been appreciated that these TLRs and the TLR coreceptor, $\mathrm{CD} 14$, participate in the response of microglia to fibrillar (or possibly oligomeric) forms of $\mathrm{A} \beta$. The significance of these latter findings is that fibrillar forms of $\mathrm{A} \beta$ (fA $\beta$ ) act as a bona fide immune stimulus to the cells through classical host-defense mechanisms. The TLRs functionally interact with other cell surface receptors, including CD36, $\alpha 6 \beta 1$ integrin, CD47 
and scavenger receptor $A$, that bind to $A \beta$ to initiate the activation of intracellular signaling pathways (Bamberger et al., 2003; Reed-Geaghan et al., 2009). This receptor ensemble mediates the activation of multiple parallel signaling cascades that are responsible for the complex cellular response to fA $\beta$. The recognition that TLRs participate in this receptor complex provides a mechanistic explanation for the ability of $\mathrm{fA} \beta$ to elicit the production and secretion of proinflammatory (Th1-type) cytokines (Mantovani et al., 2004) and production of reactive oxygen and nitrogen radicals, which typify the acquisition of a 'classical' activation phenotype (also termed an M1 phenotype, see below).

It has only recently been appreciated that TLRs participate in regulating microglial phenotypes and responses in the AD brain (Landreth and Reed-Geaghan, 2009). Examination of TLR expression in the brain revealed that there is increased expression of CD14, TLR2, and TLR4 in the AD brain and in animal models of $\mathrm{AD}$, as well as AD patients (Fassbender et al., 2004; Letiembre et al., 2007; Liu et al., 2005a; Walter et al., 2007). Plaque associated microglia exhibit elevated mRNA levels for TLR2/4/5/7/9 (Frank et al., 2009). Indeed, injection of A $\beta$ into the hippocampus provokes TLR2 gene expression (Richard et al., 2008). It is of interest that a polymorphism in TLR4 that results in a blunted signaling response corresponds to a 2.7fold reduction in risk for late onset AD (Minoretti et al., 2006).

A physical interaction between the TLR coreceptor CD14 and A $\beta$ has been demonstrated through a variety of approaches, including co-incubation of $\mathrm{fA} \beta$ and recombinant CD14, realtime surface plasmon resonance spectroscopy, and FLIM-based FRET and antibody-mediated inhibition of the interaction (Reed-Geaghan et al., 2009) There is evidence that TLRs are involved in the microglial response to A $\beta$ as inhibition of TLR4 or TLR2 through function blocking antibodies or siRNA knock-down also prevented fA $\beta$-induced nitrite, IL-6, and TNF$\alpha$ production (Jana et al., 2008; Udan et al., 2008; Walter et al., 2007). A recent study demonstrated a requirement of CD14, TLR 2 and 4 for fA $\beta$-stimulated microglial responses (Reed-Geaghan et al., 2009). A mouse model of AD lacking functional TLR4 exhibited increased levels of the microglial marker CD11b and the reactive astrocyte marker GFAP (Jin et al., 2008). In addition, AD mice deficient for TLR 2 were shown to have increased TGF $\beta$ mRNA levels in plaque associated microglia compared to wild type littermates (Richard et al., 2008). Treatment of microglia with TLR2, TLR4, or TLR9 ligands stimulates A $\beta$ uptake in vitro (Tahara et al., 2006). However, this study did not discriminate whether this was due to phagocytosis or through other internalization mechanisms (Mandrekar et al., 2009) and this issue is of importance given that the internalized $A \beta$ was found to be present in a spectrum of oligomer species, from monomers to very high MW forms. It should be noted that it has been reported that TLR9 activation in the presence of $\mathrm{A} \beta$ suppressed NO and TNF $\alpha$ release, whereas TLR2 and TLR4 ligands produced the opposite response (Lotz et al., 2005)

\section{Role of TLRs in A $\beta$ clearance}

The TLRs and the coreceptor CD14 have been implicated in A $\beta$ internalization by microglia (Liu et al., 2005b). Microglia require CD14 for phagocytic uptake of fA $\beta$ (Reed-Geaghan et al., 2009). Microglia exhibit the colocalization of CD14 and fibrillar $A \beta_{42}$ at the cell surface, and they are then internalized and trafficked to the lysosome. Microglia lacking CD14 phagocytosed less fibrillar $\mathrm{A} \beta_{42}$ than did their wild-type counterparts, though this was not due to general impairment of the phagocytic machinery. Both TLR2 and 4 are necessary for fAbstimulated phagocytosis (Reed-Geaghan et al., 2009).

The importance of TLR 4 and TLR2 in A $\beta$ uptake has also been evaluated in animal models of AD. The APPswe/PSEN1dE9 mouse model of Alzheimer's disease (Jankowsky et al., 2004) is on a mixed genetic background which includes the $\mathrm{C} 3 \mathrm{H} / \mathrm{HeJ}$ strain. These animals possess a destructive point mutation in the TLR4 gene that prevents signaling from the mutant receptor (Poltorak et al., 1998). Tahara et al. employed this model to evaluate the role of TLR4 in 
amyloidogenesis in vivo (Tahara et al., 2006). APPswe/PSENdE9 mice with inactive TLR4 exhibited increased cortical and hippocampal $\mathrm{A} \beta$ load when compared with mice with an intact TLR4 gene. These authors argued that the change in $A \beta$ load was due to a change in microglialmediated A $\beta$ clearance that was reliant upon TLR4 function. In contrast, APPswe/PSEN1dE9 mice have also been mated to TLR2 ${ }^{-/-}$mice, and the resulting transgenic TLR2 ${ }^{-/-}$mice showed delayed $A \beta$ deposition through 6 months of age. Whether this is reflective of the roles of TLRs in $A \beta$ clearance is unclear. It is noteworthy that the TLR 2 null mice had comparable deposition by 9 months of age, compared to their TLR $2^{+/+}$littermates (Richard et al., 2008). The different results in A $\beta$ deposition between TLR 4 and TLR 2 deficient animals could be explained by the age of the animals assessed. Jin et al. used mice that were aged at least 14 months, while Richard et al. examined their animals no later than 9 months of age. Hickman and colleagues have reported that the APPswe/PSEN1dE9 mouse has decreased expression of various A $\beta$-binding receptors and degrading enzymes at 8 months of age that accompanies an increase in proinflammatory gene expression (Hickman et al., 2008). It is possible that microglia can efficiently clear $A \beta$ in early stages of $A D$, however, at later stages in the disease the genes involved in $A \beta$ clearance are down-regulated contributing to $A \beta$ accumulation (Hickman et al., 2008).

Additional evidence for the roles of TLRs in A $\beta$ clearance has been provided by Herber and colleagues who reported that intrahippocampal injection of the TLR4 ligand LPS into an animal model of $\mathrm{AD}$ resulted in clearance of diffuse, but not compact, $\mathrm{A} \beta$ plaques that were associated with microglial activation (Herber et al., 2006; Herber et al., 2004). Peripheral LPS administration resulted in cerebral microglial activation and a reduction in $\mathrm{A} \beta$ plaque burden (Quinn et al., 2003), however, it has also been reported that this treatment is associated with increased $A \beta$ peptide levels (Sheng et al., 2003).

TLRs in Alzheimer's disease have generally been thought to be necessary for recognition and clearance of the higher molecular weight $A \beta$ species. TLRs evolved as a mechanism to recognize conserved PAMPs and DAMPs, and it has generally been thought that the monomeric and smaller oligomeric species of $A \beta$ were unable to be recognized by these receptors. Recently Scholtzova and colleagues demonstrated that activation of TLR 9 by methyl $\mathrm{CpG}$ leads to a reduction in oligomeric A $\beta$ species (Scholtzova et al., 2009). This still only demonstrates that activation of microglia through TLRs can lower the $A \beta$ load, and not that the oligomers themselves engage any specific receptor.

One of the principal unresolved questions in understanding the roles of microglia in the AD brain is why these cells fail to phagocytose and clear amyloid deposits in the brain. The literature is repleat with demonstrations of the phagocytic capapcity of these cells in vitro and immunotherapy studies have demonstrated that they can efficiently remove amyloid plaques from the brain when appropriately stimulated, likely through FcRs. At the moment there is no clear understanding of why microglia fail to perform this basic function. Koenigsknecht et al. reported that proinflammatory cytokine exposure was sufficient to functionally inactivate the phagocytic machinery (Koenigsknecht-Talboo and Landreth, 2005). It remains unclear whether TLR actions on phagocytosis are selectively impaired and more work in this area is needed.

\section{Alternative activation states of microglia}

The microglial response to amyloid deposition in the brain has routinely been characterized as conversion of a 'resting or quiescent' phenotype into an 'activated, proinflammatory' phenotype. The latter phenotype has been linked with neurotoxicity and other deleterious effects in the brain, mostly coordinated through cytokines (Bodles and Barger, 2004). In the past few years it has been appreciated that the response of microglia is more nuanced and 
complex than this dichotomy suggests (Colton, 2009). Moreover, the focus has been largely on the proinflammatory actions of the microglia and the vast majority of the literature documents these classical activation phenomena. Less attention has been paid to the resolution phase of the inflammatory response that engages mechanisms responsible for tissue maintenance and repair. The failure to appreciate the coordinated nature of the microglia/ macrophage response whereby microglial activation is followed by induction of tissue repair mechanisms has contributed to the confusion over whether "inflammation" has positive or negative effects in the AD brain and other CNS disorders (Hanisch and Kettenmann, 2007; Wyss-Coray and Mucke, 2002).

The classical activation of these cells is inferred by the induction of a number of cell surface proteins, most prominently receptors that participate in the innate immune response. The engagement of these host defense mechanisms, while effective in pathogen inactivation, produces collateral damage in the surrounding tissue. The classical activation is accompanied by the elaboration of chemokines, notably CCL2 (MCP-1), which mediate the recruitment and migration of microglia to amyloid deposits (El Khoury et al., 2007), where they invest the plaque with their processes. It is noteworthy that in the $\mathrm{AD}$ brain such classically activated microglia are associated with plaques throughout the disease process and are responsible for the proinflammatory milieu in the AD brain. Also, after exposure to amyloid, microglia are unable to express class II major histocompatibility complex (MHC-II) which would be important in regulating any $\mathrm{T}$-cell mediated response that occurs during the disease course (Butovsky et al., 2005; Town et al., 2005)

Microglia and other tissue macrophages exhibit a sophisticated and coordinated response through which the cells inactivate the initial immune response and initiate genetic programs that mediate tissue repair (Martinez et al., 2009). These mechanisms are associated with the production of anti-inflammatory cytokines. Most prominent amongst these are IL-4, IL-10, IL-13 and TGF $\beta$ which are produced by both glia and neurons and are associated with the conversion to other phenotypes, see Figure 1 (Colton, 2009). These anti-inflammatory (Th2type) cytokines are central to the development of what has been termed an "alternatively activated" state (also called M2) (Mantovani et al., 2004). However, the order, duration, and combinations in which microglia are exposed to stimulants may prove to be crucial in determining their response (Schwartz et al., 2006; Stout et al., 2005). Alternatively activated macrophages are characterized by the suppression of Th1-proinflammatory cytokines, reduced NOS 2 expression, NOX2 activation and induction of a number of genes that are characteristic on the acquisition of this phenotype. The conversion to an alternative activation state appears to be coordinated by two members of the peroxisome proliferator-activated receptor family, PPAR $\gamma$ and PPAR $\beta / \delta$. (Kang et al., 2008; Odegaard and Chawla, 2008; Odegaard et al., 2007; Odegaard et al., 2008) which act to regulate the program of gene expression that characterizes this macrophage phenotype (Gallardo-Soler et al., 2008). IL-4 receptor activation stimulates the generation of endogenous PPAR $\gamma$ ligands (Martinez et al., 2008) and the $\operatorname{PPAR} \gamma$ gene. There is reciprocal response in which PPAR $\gamma$ activation in the brain is reported to result in elevated IL-4 levels (Loane et al., 2009).

Alternatively activated murine macrophages express genes such as arginase 1, YM1, YM2, FIZZ1 (RELM $\alpha$ ) and the mannose receptor (CD206) (Martinez et al., 2009). Indeed, IL-4 treatment of microglia stimulates the expression of these genes (Colton et al., 2006). This activation state has been termed M2. Colton and colleagues reported that these genes were also expressed at elevated levels in Tg2576 mice, a mouse model of AD, and in humans with AD. (Colton et al., 2006). Maier et al. found that in aged hAPP expressing mice lacking the complement factor $\mathrm{C} 3$ the in the brain the microglia exhibited an alternative activation phenotype, as evidenced by the reduced expression of NOS2 (Maier et al., 2008). These C3deficient animals had increased IL-4 and IL-10 levels compared to those with the wild type 
allele. Also, IL-4 treatment of M2 microglia has been shown to lead to preferential uptake and degradation of $A \beta$ dimers and tetramers in vitro (Shimizu et al., 2008). Analysis of aged APP/ PS1 mice by Jimenez and colleagues has yielded somewhat different results and interpretations (Jimenez et al., 2008). Examination of young animals found that microglia exhibited little expression of inflammatory cytokines, but YM1 expression was induced and marker expressing microglia were associated with plaques. In older animals, there was increased expression of inflammatory cytokines and NOS2, and these cells retained their expression of YM1, leading to the conclusion that the microglial phenotype changed from alternative to classic activation in this $\mathrm{AD}$ model. However, it is possible that the microglial population is functionally heterogeneous (see below).

A more detailed analysis of macrophage phenotypes has led to the identification of two distinct alternative activation states (Mantovani et al., 2004). The anti-inflammatory M2a state described above reflects the actions of IL-4 and IL-13. However, the anti-inflammatory actions elicited by IL-10 and TGF $\beta$ have a similar, but qualitatively and quantitatively distinct range of effects. This phenotype is termed 'acquired deactivation' or M2c and is associated with the robust suppression of the innate immune response, refer to Figure 1. The acquired deactivation response is also elicited by exposure of macrophages to apoptotic cells. This is a highly evolved cellular response that allows the macrophage to detect apoptotic cells and ingest them without inducing a classical innate immune response. This allows normal developmental processes and accompanying patterned cell death as well as normal tissue maintenance to be performed without setting off an inflammatory response. Consequently, macrophages have evolved a number of mechanisms through which they can use receptors to detect cell surface phosphatidylserine molecules, either directly or through adapter proteins, which are uniquely found on apoptotic cells (Grommes et al., 2008). Engagement of these receptors simultaneously acts to suppress the activity of promoters of inflammatory genes, stimulate the expression of the anti-inflammatory cytokines IL-10 and TGF $\beta$ and stimulate phagocytosis, allowing removal of the cellular corpse from the tissue. There is recent evidence that the microglial triggering receptor expressed by myeloid cells 2 , TREM2, plays analogous roles. TREM2 interacts with an undefined neuronal cell surface protein and this ligand-receptor interaction stimulates the uptake of apoptotic neurons and suppresses microglia inflammatory cytokine expression (Hsieh et al., 2009). TREM2 is expressed at elevated levels in an animal model of AD in plaque-associated microglia (Frank et al., 2008).

\section{Infiltration of the brain by peripherally-derived monocytes}

There is a general consensus that endogenous microglia mediate a local inflammatory response upon exposure to fA $\beta$ and amyloid deposition in the AD brain and its murine models. However, this has recently been challenged by a number of recent studies that provide evidence that the brain is invaded by peripherally-derived monocytes or macrophages (Rezai-Zadeh et al., 2009). The outcomes of these studies are provocative, and have generated substantial controversy owing to a lack of agreement over the identification of peripherally derived monocytic cells and experimental artifacts in some studies.

In the normal mature brain the turnover of microglia is low and these cells are renewed by proliferation of endogenous microglia (Ajami et al., 2007; Chan et al., 2007; Mildner et al., 2007). Infiltration of peripheral blood cells is normally prevented by the BBB. Specifically, the infiltration of peripheral monocytes into the normal brain is infrequent and the levels of these cells are very low. A number of CNS disorders, brain injury or systemic infections (D'Mello et al., 2009) are accompanied by infiltration of the brain by peripheral moncytes, macrophages or T-lymphocytes. These cells migrate under the influence of cytokines and chemokines, most prominently CCL2. The role of invading peripherally derived monocytes in Alzheimer's disease has recently received considerable attention and generated substantial 
controversy. Simard et al. reported that in the brains murine models of AD were infiltrated by significant numbers of bone marrow derived macrophages (Simard et al., 2006). These cells were found to be associated with amyloid plaques and were able to effectively phagocytose and clear A $\beta$ deposits from the brain. Significantly, selective elimination of these cells by genetic and pharmacological manipulations resulted in increased plaque burden. These data were interpreted as evidence that the bone marrow derived monocytes had a range of behaviors that distinguished them from resident microglia and raised the prospect that genetic manipulation of bone marrow progenitors might allow new therapeutic approaches to $\mathrm{AD}$ (Rivest, 2009). These experiments involved the use of chimeric mice in which the host animal was irradiated and the bone marrow replaced with that of transgenic mice in which lineage markers or selection genes had been introduced. The host/transplant methodology has a number of confounds that were argued to have invalidated the conclusions from this study (Ransohoff, 2007). In a pair of complementary papers, Ajami et al. (Ajami et al., 2007) and Mildner et al. (Mildner et al., 2007) reported that the infiltration of the bone marrow derived cells into the brain was an artifact of the chimeric host/transplant methodology. Indeed, studies using irradiated mice with transplanted fluorescently labeled bone marrow progenitors provided much of the rationale for this hypothesis (Malm et al., 2005; Stalder et al., 2005).

More compelling evidence was provided by studies of El Khoury and colleagues (El Khoury et al., 2007) who reported the effects of knockout of the chemokine Ccl2/MCP-1 receptor, $C c r 2$, in an animal model of AD. Myeloid lineage cells expressing this receptor migrate in response to the chemokine that is expressed at the site of injury, or in the case of $\mathrm{AD}$, by plaque associated microglia and astrocytes, thus accounting for the recruitment of microglia to these deposits. These authors proposed that this signaling system might be responsible for stimulating the infiltration of the brain by monocytes or macrophages from the peripheral circulation or by perivascular macrophages. Loss of Ccr2 expression was associated with many fewer microglia in the brain and increased $A \beta$ levels. They argue that the increased numbers of monocytes/microglia in the brains of APP expressing mice was due to infiltration of peripherally derived cells, based on the abundance of cells expressing high levels of CD45, a characteristic of this population that distinguishes them from endogenous microglia which have lower levels of CD45 expression. The magnitude of the effect was impressive with an approximate 8 fold increase in CD45 hi cells (from $1 \%$ to $8 \%$ of total CD45 positive cells in the brain) and consistent with an overall 12 fold increase in the total number of microglia in the Tg2576 brain. However, the loss of $C c r 2$ in the APP expressing mice was associated with microglia numbers roughly similar to that of wild type mice, owing to the impaired chemotaxis/ migration of macrophages/monocytes. Town and colleagues have provided additional support for the notion that bone marrow derived monocytes/macrophages immigrate into the parenchyma of the brain of animal models of AD and ameliorate AD-like pathology (Town et al., 2008). These authors expressed a dominant negative form of the TGF $\beta$ receptor using a $\mathrm{CD} 11 \mathrm{c}$ promoter and expressed in myeloid lineage cells. Contrary to their expectations, plaque pathology was dramatically less severe and behavior was improved in aged APP-expressing Tg2576 mice when TGF $\beta$ signaling was suppressed. Significantly, these latter mice exhibited increased numbers of plaque-related cells expressing CD $45^{\text {hi }}$ and other macrophage markers. The authors argue that the increase in CD45 hi cells reflects the infiltration of the brain by bloodderived macrophages. However, they did not detect significant expression of the TGF $\beta$ receptor transgene in the brain and this is a potential confound in interpretation of the data given the magnitude of the increase in CD45 hi expressing cells.

The interpretation of both of these studies is reliant upon the fidelity of the markers employed. While the conclusions are reasonable if CD 45 and CD11c expression is restricted to infiltrating cells and is unaltered for a significant period of time once the cells are resident in the tissue. Both of these genes are subject to acute regulation in this lineage and thus there remains a degree of uncertainty about the origin of these cells. Indeed, a subset of endogenous microglia 
have recently been reported to express $\mathrm{CD} 11 \mathrm{c}$, a canonical marker of dendritic cells (Bulloch et al., 2008). It will be important to establish exactly how many cells immigrate into the brain parenchyma, and the kinetics of this process. Also, it is of interest to know how long they remain in the tissue, whether they are trafficked out of the tissue or undergo apoptosis upon clearance of amyloid deposits and whether they exhibit sustained expression of the identifying markers.

\section{Conclusions}

As we have discussed, the response of microglia can vary along a spectrum of different states and some can be detrimental. Major questions remaining for the field, while not new, include whether or not peripheral monocytes are able to infiltrate the brain and alter the disease course in $\mathrm{AD}$. Also, it will be important to determine whether the activation state of the resident microglia is different from the infiltrating monocytes. Furthermore, are there differentially activated microglia around the same plaques? Also, does the activation status of the microglia alter during the disease course and does their adaptability lessen as the organism ages?

Therefore, the challenge in the development of future treatments will be not simply turning on or off the immune response, but tuning the dial so that the protective effects are augmented and the negative effectives dampened.

\section{Acknowledgments}

This work was supported by grants from the NIA (AG16740 and AG030482), the Alzheimer Association and from the Blanchette Hooker Rockefeller Foundation.

\section{References}

Adams S, et al. Signal-regulatory protein is selectively expressed by myeloid and neuronal cells. J Immunol 1998;161:1853-9. [PubMed: 9712053]

Ajami B, et al. Local self-renewal can sustain CNS microglia maintenance and function throughout adult life. Nat Neurosci 2007;10:1538-43. [PubMed: 18026097]

Akiyama H, et al. Inflammation and Alzheimer's disease. Neurobiology of Aging 2000;21:383-421. [PubMed: 10858586]

Bamberger ME, et al. A cell surface receptor complex for fibrillar beta-amyloid mediates microglial activation. J Neurosci 2003;23:2665-74. [PubMed: 12684452]

Bianchi ME. DAMPs, PAMPs and alarmins: all we need to know about danger. J Leukoc Biol 2007;81:15. [PubMed: 17032697]

Bodles AM, Barger SW. Cytokines and the aging brain - what we don't know might help us. Trends Neurosci 2004;27:621-6. [PubMed: 15374674]

Bornemann KD, et al. Abeta-induced inflammatory processes in microglia cells of APP23 transgenic mice. Am J Pathol 2001;158:63-73. [PubMed: 11141480]

Bulloch K, et al. CD11c/EYFP transgene illuminates a discrete network of dendritic cells within the embryonic, neonatal, adult, and injured mouse brain. J Comp Neurol 2008;508:687-710. [PubMed: 18386786]

Butovsky O, et al. Activation of microglia by aggregated beta-amyloid or lipopolysaccharide impairs MHC-II expression and renders them cytotoxic whereas IFN-gamma and IL-4 render them protective. Mol Cell Neurosci 2005;29:381-93. [PubMed: 15890528]

Cagnin A, et al. Positron emission tomography imaging of neuroinflammation. Neurotherapeutics 2007;4:443-52. [PubMed: 17599710]

Cardona AE, et al. Control of microglial neurotoxicity by the fractalkine receptor. Nat Neurosci 2006;9:917-24. [PubMed: 16732273]

Chan WY, et al. The origin and cell lineage of microglia: new concepts. Brain Res Rev 2007;53:344-54. [PubMed: 17188751] 
Colton C. Heterogeneity of Microglial Activation in the Innate Immune Response in the Brain. J Neuroimmune Pharmacol. 2009

Colton CA, et al. Expression profiles for macrophage alternative activation genes in AD and in mouse models of AD. J Neuroinflammation 2006;3:27. [PubMed: 17005052]

Combs C. Inflammation and Microglia Actions in Alzheimer's Disease. J Neuroimmune Pharmacol. 2009

D'Mello C, et al. Cerebral microglia recruit monocytes into the brain in response to tumor necrosis factoralpha signaling during peripheral organ inflammation. J Neurosci 2009;29:2089-102. [PubMed: 19228962]

Davalos D, et al. ATP mediates rapid microglial response to local brain injury in vivo. Nat Neurosci 2005;8:752-8. [PubMed: 15895084]

Doorduin J, et al. [(11)C]-DPA-713 and [(18)F]-DPA-714 as New PET Tracers for TSPO: A Comparison with [(11)C]-(R)-PK11195 in a Rat Model of Herpes Encephalitis. Mol Imaging Biol. 2009

Edison P, et al. Microglia, amyloid, and cognition in Alzheimer's disease: An [11C](R)PK11195-PET and [11C]PIB-PET study. Neurobiol Dis 2008;32:412-9. [PubMed: 18786637]

El Khoury J, et al. Ccr2 deficiency impairs microglial accumulation and accelerates progression of Alzheimer-like disease. Nat Med 2007;13:432-8. [PubMed: 17351623]

Fassbender K, et al. The LPS receptor (CD14) links innate immunity with Alzheimer's disease. Faseb J 2004;18:203-5. [PubMed: 14597556]

Floden AM, Combs CK. Beta-amyloid stimulates murine postnatal and adult microglia cultures in a unique manner. J Neurosci 2006;26:4644-8. [PubMed: 16641245]

Frank S, et al. TREM2 is upregulated in amyloid plaque-associated microglia in aged APP23 transgenic mice. Glia 2008;56:1438-47. [PubMed: 18551625]

Frank S, et al. Differential regulation of toll-like receptor mRNAs in amyloid plaque-associated brain tissue of aged APP23 transgenic mice. Neurosci Lett 2009;453:41-4. [PubMed: 19429012]

Gallardo-Soler A, et al. Arginase I induction by modified lipoproteins in macrophages: a peroxisome proliferator-activated receptor-gamma/delta-mediated effect that links lipid metabolism and immunity. Mol Endocrinol 2008;22:1394-402. [PubMed: 18323470]

Grommes C, et al. Regulation of microglial phagocytosis and inflammatory gene expression by Gas 6 acting on the Axl/Mer family of tyrosine kinases. J Neuroimmune Pharmacol 2008;3:130-40. [PubMed: 18247125]

Hanisch U-K, Kettenmann H. Microglia: active sensor and versatile effector cells in the normal and pathologic brain. Nat Neurosci 2007;10:1387-94. [PubMed: 17965659]

Herber DL, et al. Diverse microglial responses after intrahippocampal administration of lipopolysaccharide. Glia 2006;53:382-91. [PubMed: 16288481]

Herber DL, et al. Time-dependent reduction in Abeta levels after intracranial LPS administration in APP transgenic mice. Exp Neurol 2004;190:245-53. [PubMed: 15473997]

Hickman SE, et al. Microglial dysfunction and defective beta-amyloid clearance pathways in aging Alzheimer's disease mice. J Neurosci 2008;28:8354-60. [PubMed: 18701698]

Hsieh CL, et al. A role for TREM2 ligands in the phagocytosis of apoptotic neuronal cells by microglia. J Neurochem 2009;109:1144-56. [PubMed: 19302484]

Jana M, et al. Fibrillar amyloid-beta peptides activate microglia via TLR2: implications for Alzheimer's disease. J Immunol 2008;181:7254-62. [PubMed: 18981147]

Jankowsky J, et al. Mutant presenilins specifically elevate levels of the 42 residue beta-amyloid peptide in vivo: evidence for augmentation of a 42-specific gamma secretase. Human Molecular Genetics 2004;13:159-170. [PubMed: 14645205]

$\mathrm{Ji} \mathrm{B}$, et al. Imaging of peripheral benzodiazepine receptor expression as biomarkers of detrimental versus beneficial glial responses in mouse models of Alzheimer's and other CNS pathologies. J Neurosci 2008;28:12255-67. [PubMed: 19020019]

Jimenez S, et al. Inflammatory response in the hippocampus of PS1M146L/APP751SL mouse model of Alzheimer's disease: age-dependent switch in the microglial phenotype from alternative to classic. $\mathrm{J}$ Neurosci 2008;28:11650-61. [PubMed: 18987201]

Jin JJ, et al. Toll-like receptor 4-dependent upregulation of cytokines in a transgenic mouse model of Alzheimer's disease. J Neuroinflammation 2008;5:23. [PubMed: 18510752] 
Kang K, et al. Adipocyte-derived Th2 cytokines and myeloid PPARdelta regulate macrophage polarization and insulin sensitivity. Cell Metab 2008;7:485-95. [PubMed: 18522830]

Koenigsknecht-Talboo J, Landreth GE. Microglial phagocytosis induced by fibrillar beta-amyloid and IgGs are differentially regulated by proinflammatory cytokines. J Neurosci 2005;25:8240-9. [PubMed: 16148231]

Kusakari S, et al. Trans-endocytosis of CD47 and SHPS-1 and its role in regulation of the CD47-SHPS-1 system. J Cell Sci 2008;121:1213-23. [PubMed: 18349073]

Landreth GE, Reed-Geaghan EG. Toll-like receptors in Alzheimer's disease. Curr Top Microbiol Immunol 2009;336:137-53. [PubMed: 19688332]

Letiembre M, et al. Screening of innate immune receptors in neurodegenerative diseases: A similar pattern. Neurobiol Aging. 2007

Liu Y, et al. LPS receptor (CD14): a receptor for phagocytosis of Alzheimer's amyloid peptide. Brain 2005a;128:1778-89. [PubMed: 15857927]

Liu Y, et al. LPS receptor (CD14): a receptor for phagocytosis of Alzheimer's amyloid peptide. Brain 2005b;128:1778-1789. [PubMed: 15857927]

Loane DJ, et al. Interleukin-4 mediates the neuroprotective effects of rosiglitazone in the aged brain. Neurobiol Aging 2009;30:920-31. [PubMed: 17950491]

Lotz M, et al. Amyloid beta peptide 1-40 enhances action of Toll like receptor-2 and -4 agonists but antagonizes Toll-like receptor-9-induced inflammation in primary mouse microglial cell cultures. Journal of Neurochemistry 2005;94:289-298. [PubMed: 15998280]

Lyons A, et al. CD200 ligand receptor interaction modulates microglial activation in vivo and in vitro: a role for IL-4. J Neurosci 2007;27:8309-13. [PubMed: 17670977]

Maeda J, et al. Longitudinal, quantitative assessment of amyloid, neuroinflammation, and anti-amyloid treatment in a living mouse model of Alzheimer's disease enabled by positron emission tomography. J Neurosci 2007;27:10957-68. [PubMed: 17928437]

Maier M, et al. Complement C3 deficiency leads to accelerated amyloid beta plaque deposition and neurodegeneration and modulation of the microglia/macrophage phenotype in amyloid precursor protein transgenic mice. J Neurosci 2008;28:6333-41. [PubMed: 18562603]

Malm TM, et al. Bone-marrow-derived cells contribute to the recruitment of microglial cells in response to beta-amyloid deposition in APP/PS1 double transgenic Alzheimer mice. Neurobiol Dis 2005;18:134-42. [PubMed: 15649704]

Mandrekar S, et al. Microglia mediate the clearance of soluble Abeta through fluid phase macropinocytosis. J Neurosci 2009;29:4252-62. [PubMed: 19339619]

Mantovani A, et al. The chemokine system in diverse forms of macrophage activation and polarization. Trends Immunol 2004;25:677-86. [PubMed: 15530839]

Martinez FO, et al. Alternative activation of macrophages: an immunologic functional perspective. Annu Rev Immunol 2009;27:451-83. [PubMed: 19105661]

Martinez FO, et al. Macrophage activation and polarization. Front Biosci 2008;13:453-61. [PubMed: 17981560]

Matozaki T, et al. Functions and molecular mechanisms of the CD47-SIRPalpha signalling pathway. Trends Cell Biol 2009;19:72-80. [PubMed: 19144521]

Meyer-Luehmann M, et al. Rapid appearance and local toxicity of amyloid-beta plaques in a mouse model of Alzheimer's disease. Nature 2008;451:720-4. [PubMed: 18256671]

Mildner A, et al. Microglia in the adult brain arise from Ly-6ChiCCR2+ monocytes only under defined host conditions. Nat Neurosci 2007;10:1544-53. [PubMed: 18026096]

Minoretti P, et al. Effect of the functional toll-like receptor 4 Asp299Gly polymorphism on susceptibility of late-onset Alzheimer's disease. Neuroscience Letters 2006;391:147-149. [PubMed: 16157451]

Nimmerjahn A, et al. Resting microglial cells are highly dynamic surveillants of brain parenchyma in vivo. Science 2005;308:1314-8. [PubMed: 15831717]

Odegaard JI, Chawla A. Mechanisms of macrophage activation in obesity-induced insulin resistance. Nat Clin Pract Endocrinol Metab 2008;4:619-26. [PubMed: 18838972]

Odegaard JI, et al. Macrophage-specific PPARgamma controls alternative activation and improves insulin resistance. Nature 2007;447:1116-20. [PubMed: 17515919] 
Odegaard JI, et al. Alternative M2 activation of Kupffer cells by PPARdelta ameliorates obesity-induced insulin resistance. Cell Metab 2008;7:496-507. [PubMed: 18522831]

Okello A, et al. Microglial activation and amyloid deposition in mild cognitive impairment: a PET study. Neurology 2009;72:56-62. [PubMed: 19122031]

Palm NW, Medzhitov R. Pattern recognition receptors and control of adaptive immunity. Immunol Rev 2009;227:221-33. [PubMed: 19120487]

Poltorak A, et al. Defective LPS signaling in C3H/HeJ and C57BL/10ScCr mice: mutations in TLR4 gene. Science 1998;282:2085-2088. [PubMed: 9851930]

Quinn J, et al. Inflammation and cerebral amyloidosis are disconnected in an animal model of Alzheimer's disease. J Neuroimmunol 2003;137:32-41. [PubMed: 12667645]

Ransohoff RM. Microgliosis: the questions shape the answers. Nat Neurosci 2007;10:1507-9. [PubMed: 18043584]

Ransohoff RM, et al. Chemokines and chemokine receptors: multipurpose players in neuroinflammation. Int Rev Neurobiol 2007;82:187-204. [PubMed: 17678962]

Ransohoff RM, Perry VH. Microglial physiology: unique stimuli, specialized responses. Annu Rev Immunol 2009;27:119-45. [PubMed: 19302036]

Reed-Geaghan EG, et al. CD14 and Toll-Like Receptors 2 and 4 Are Required for Fibrillar A \{beta $\}$ Stimulated Microglial Activation. Journal of Neuroscience 2009;29:11982. [PubMed: 19776284]

Rezai-Zadeh K, et al. CNS Infiltration of Peripheral Immune Cells: D-Day for Neurodegenerative Disease? J Neuroimmune Pharmacol. 2009

Richard KL, et al. Toll-like receptor 2 acts as a natural innate immune receptor to clear amyloid beta 1-42 and delay the cognitive decline in a mouse model of Alzheimer's disease. J Neurosci 2008;28:578493. [PubMed: 18509040]

Rivest S. Regulation of innate immune responses in the brain. Nat Rev Immunol 2009;9:429-39. [PubMed: 19461673]

Scholtzova $\mathrm{H}$, et al. Induction of toll-like receptor 9 signaling as a method for ameliorating Alzheimer's disease-related pathology. J Neurosci 2009;29:1846-54. [PubMed: 19211891]

Schwartz M, et al. Microglial phenotype: is the commitment reversible? Trends Neurosci 2006;29:6874. [PubMed: 16406093]

Sheng JG, et al. Lipopolysaccharide-induced-neuroinflammation increases intracellular accumulation of amyloid precursor protein and amyloid beta peptide in APPswe transgenic mice. Neurobiol Dis 2003;14:133-45. [PubMed: 13678674]

Shimizu E, et al. IL-4-induced selective clearance of oligomeric beta-amyloid peptide(1-42) by rat primary type 2 microglia. J Immunol 2008;181:6503-13. [PubMed: 18941241]

Simard AR, et al. Bone marrow-derived microglia play a critical role in restricting senile plaque formation in Alzheimer's disease. Neuron 2006;49:489-502. [PubMed: 16476660]

Stalder AK, et al. Invasion of hematopoietic cells into the brain of amyloid precursor protein transgenic mice. J Neurosci 2005;25:11125-32. [PubMed: 16319312]

Stout RD, et al. Macrophages sequentially change their functional phenotype in response to changes in microenvironmental influences. J Immunol 2005;175:342-9. [PubMed: 15972667]

Streit WJ, et al. Microglial degeneration in the aging brain--bad news for neurons? Front Biosci 2008;13:3423-38. [PubMed: 18508444]

Streit WJ, et al. Dystrophic microglia in the aging human brain. Glia 2004;45:208-12. [PubMed: 14730714]

Tahara K, et al. Role of toll-like receptor signalling in Abeta uptake and clearance. Brain 2006;129:30063019. [PubMed: 16984903]

Town T, et al. Blocking TGF-beta-Smad2/3 innate immune signaling mitigates Alzheimer-like pathology. Nat Med 2008;14:681-7. [PubMed: 18516051]

Town T, et al. T-cells in Alzheimer's disease. NeuroMolecular Medicine 2005;7:255-64. [PubMed: 16247185]

Udan ML, et al. Toll-like receptors 2 and 4 mediate Abeta(1-42) activation of the innate immune response in a human monocytic cell line. J Neurochem 2008;104:524-33. [PubMed: 17986235] 
Wake $\mathrm{H}$, et al. Resting microglia directly monitor the functional state of synapses in vivo and determine the fate of ischemic terminals. J Neurosci 2009;29:3974-80. [PubMed: 19339593]

Walker DG, et al. Decreased expression of CD200 and CD200 receptor in Alzheimer's disease: a potential mechanism leading to chronic inflammation. Exp Neurol 2009;215:5-19. [PubMed: 18938162]

Walter S, et al. Role of the toll-like receptor 4 in neuroinflammation in Alzheimer's disease. Cell Physiol Biochem 2007;20:947-56. [PubMed: 17982277]

Wiley CA, et al. Carbon 11-labeled Pittsburgh Compound B and carbon 11-labeled (R)-PK11195 positron emission tomographic imaging in Alzheimer disease. Arch Neurol 2009;66:60-7. [PubMed: 19139300]

Wyss-Coray T, Mucke L. Inflammation in neurodegenerative disease--a double-edged sword. Neuron 2002;35:419-32. [PubMed: 12165466]

Yan P, et al. Characterizing the Appearance and Growth of Amyloid Plaques in APP/PS1 Mice. J Neurosci 2009;29:10706-14. [PubMed: 19710322] 


\section{Surveying}

\section{Classical}

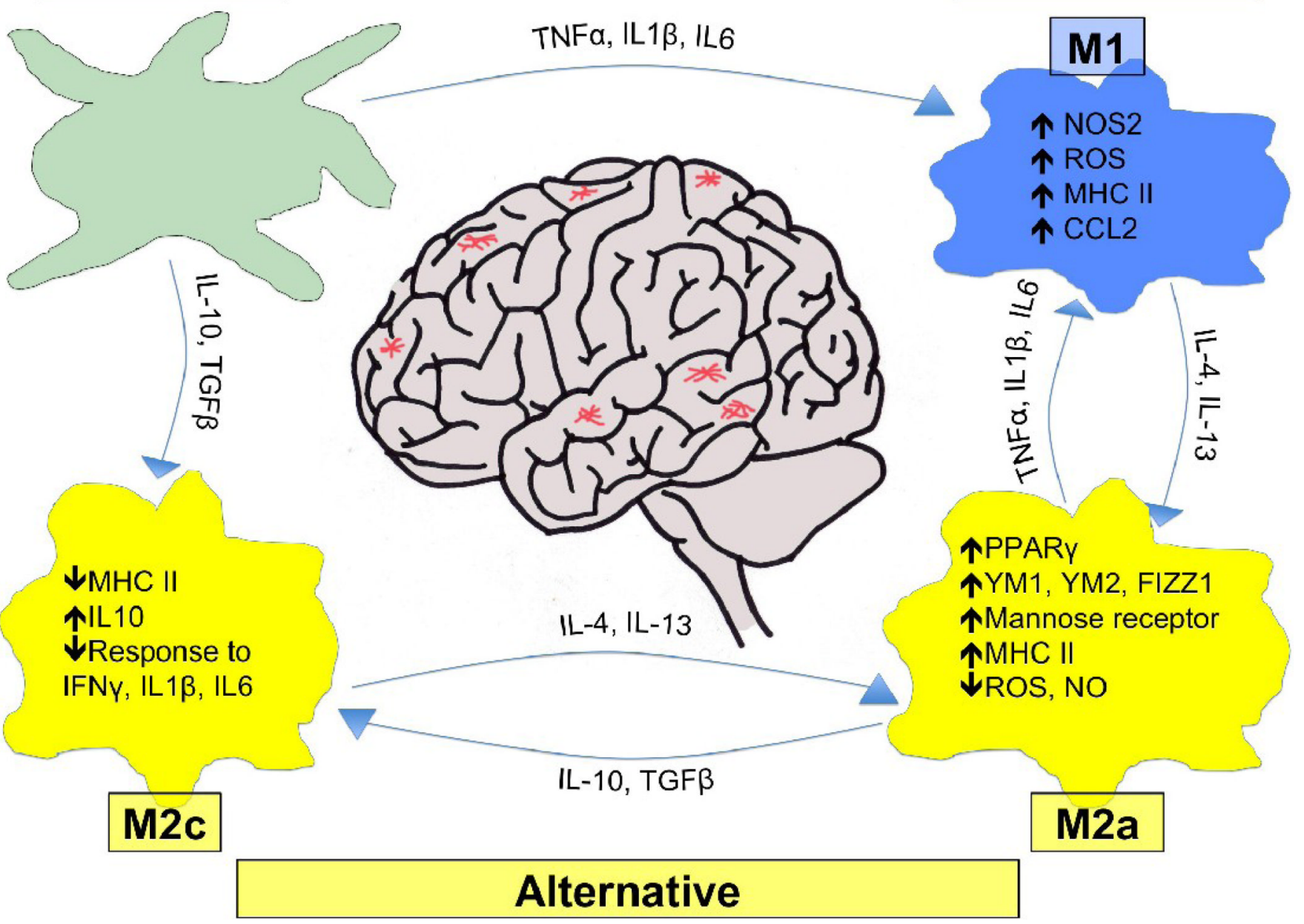

Figure 1. Different activation states of microglia

Microglia exist in different phenotypic states with differing physiological characterizations, ranging from surveying to classical to various alternatively activated states. ROS, reactive oxygen species; NO, nitric oxide; MHC II, major histocompatibility complex. 